\title{
El modelo de relaciones colectivas peruano: del intervencionista y restrictivo al promocional
}

\section{The model of peruvian collective relations: from interventionist and restrictive to promotional}

\section{ALFREDO VILLAVICENCIO*}

Resumen: El artículo parte de considerar que un sistema o modelo democrático de relaciones de trabajo implica un grado significativo de autodeterminación, mediante la presencia gravitante de la autonomía colectiva como la autorregulación pactada en las relaciones entre los representantes de los trabajadores y empleadores. Ello colisiona con el hecho de que en América Latina, con excepción de Uruguay, el modelo es marcadamente restrictivo. Si bien nuestro país desde la Constitución de 1979 se adscribe explícitamente al modelo de Estado Social de Derecho - lo que en el campo laboral se expresaba en la consagración de un modelo democrático de relaciones laborales-, esto no ha tenido correlato en la regulación infraconstitucional. Seguidamente, el artículo nos presenta las principales características de un modelo democrático de relaciones laborales que tiene como principales rasgos: i. La concepción dialéctica de las relaciones de trabajo y asignación al conflicto de un papel funcional dentro del esquema constitucional; ii. La consagración de la autonomía colectiva como pieza esencial del sistema normativo; iii. La intervención promocional y alimentadora del sistema por parte del Estado como noción de cierre de excepcional trascendencia. Finalmente, el artículo señala las principales novedades que sobre el tema contiene el Proyecto de Ley General de Trabajo, el cual se enmarca dentro del nuevo contexto latinoamericano de impulso de la autonomía colectiva que se vive en países como Brasil, Paraguay, República Dominicana y Uruguay, y que además busca impulsar la paulatina sustitución del modelo descentralizado y disfuncional para el funcionamiento del país, estableciendo una cierta preferencia por la negociación sectorial.

Palabras clave: autonomía colectiva - relaciones colectivas de trabajo negociación colectiva - modelo intervencionista - modelo democrático modelo promocional

Summary: The article begins by considering a system or democratic model of labor relations implies a significant degree of self-gravitating through the presence of collective autonomy as self-regulation agreed in relations between the representatives of workers and employers. That collides with the fact that in Latin America, with the exception of Uruguay, the model is remarkably restrictive. Although our country since the Constitutionof 1979 explicitly ascribes to the model of Social State of Law - which in the labor field was expressed in the consecration of a democratic model of labor relations, this has not had correlation with the infra-constitutional regulation.

* Doctor en Derecho por la Universidad de Sevilla, coordinador del Doctorado en Derecho de la Maestría en Relaciones Laborales y profesor de grado y posgrado de la Pontificia Universidad Católica del Perú. 
Then, the article presents the main characteristics of a democratic model of labor relations which has as main features: i.-The dialectical conception of labor relations and assignment to the conflict of a functional role within the constitutional scheme, ii.- The consecration of collective autonomy as an essential piece of the regulatory system, iii.- The promotional intervention and feeder system from the state as a notion of closure of exceptional transcendence. Finally, the article points out the principalnovelties on the subject contained in the Project of General Labor Law, the same that is in line within the new Latin American context of impulse of the collective autonomy that exists in countries like Brazil, Paraguay, Dominican Republic, Uruguay and also seeks to promote the gradual substitution of decentralized and dysfunctional model for the functioning of the country, establishing a certain preference for the sectorial bargaining.

Keywords: collective autonomy - labor collective relations - collective bargaining - interventionist model - democratic model - promotional model

\begin{abstract}
CONTENIDO: I. MARCO CONCEPTUAL GENERAL.- II. EL MODELO INTERVENCIONISTA RESTRICTIVO.- III. CUESTIONAMIENTOS JURÍDICOS Y ECONÓMICOS AL MODELO INTERVENCIONISTA EN EL PERÚ.- IV. EL MODELO DEMOCRÁTICO.- V. EL MODELO PROMOCIONAL DE RELACIONES LABORALES EN EL PROYECTO DE LEY GENERAL DEL TRABAJO.
\end{abstract}

\title{
I. MARCO CONCEPTUAL GENERAL
}

La democracia no solo es una cuestión política. Su presencia en el campo de las relaciones de trabajo es fundamental para que dé un salto del ámbito meramente formal al sustancial. Basta ver la diferencia de calidad de vida y oportunidades (a partir de la diferencia en remuneraciones y condiciones de trabajo) entre los trabajadores sujetos a un convenio colectivo y los que no lo están para reconocer la plena validez de la afirmación anterior.

Además de ello, así como la democracia política se sustenta en la voluntad popular, un sistema o modelo democrático de relaciones de trabajo importa un grado significativo de autodeterminación, mediante la presencia gravitante de la autonomía colectiva como la autorregulación pactada en las relaciones entre los representantes de los trabajadores y empleadores. En ese sentido, el fenómeno sindical es uno de los componentes básicos de la autonomía colectiva, y por tanto es un componente esencial de la democracia, que le da un rostro más humano y que canaliza el conflicto laboral de la manera más eficiente y adecuada a partir del papel preponderante de las partes en la regulación concreta (y, por tanto, adecuada) de las condiciones de trabajo, la organización del trabajo y el reparto del excedente. 
Al respecto, nuestro país posee una tradición histórica donde el protagonismo del sistema de relaciones laborales no se centra en los actores sociales, sino que pasa por la actividad estatal (normativa y administrativa, preponderantemente). Dicha tradición lo ha situado como ejemplo paradigmático del modelo latinoamericano de relaciones laborales, aunque en los últimos años se han comenzado a discutir propuestas legales que, recogiendo normas constitucionales que no solo reconocen los derechos colectivos sino que ordenan garantizarlos y promoverlos, buscan un cambio radical de modelo, apuntando hacia un modelo democrático de relaciones colectivas de trabajo, consolidando sujetos colectivos y traspasando parcelas de poder regulador de las relaciones de trabajo a quienes ostentan la condición de sujetos de las mismas. El análisis concreto de estos modelos será abordado en los siguientes capítulos, pero antes de iniciar tal labor debemos partir de establecer el marco conceptual general sobre el que se construirá nuestra reflexión.

Tratando de simplificar, diremos que se ha discutido mucho (sobre todo en la doctrina anglosajona de los años cincuenta, sesenta y setenta, y en la italiana y rioplatense de los años ochenta en adelante) respecto de la visión de las relaciones industriales como sistema. El aporte inicial determinante en este terreno fue, sin duda, el de J.T. Dunlop, que con su libro Industrial Relations System ${ }^{1}$, inauguró de manera potente esa concepción, que con variaciones, énfasis, correcciones o modificaciones, ha marcado el estudio de todo lo que gira alrededor de las relaciones entre trabajadores (y sobre todo sus organizaciones), empleadores (y sus organizaciones) y Estado. El sistema de relaciones industriales puede definirse como aquel construido a partir del surgimiento y configuración de las representaciones de trabajadores y de las relaciones entabladas entre estas y el Estado (y, en ese terreno, tenemos que decirlo, el análisis jurídico no es el predominante, sino que más bien hay un acercamiento interdisciplinario donde se combina el análisis jurídico, en menores dosis, con los enfoques económico, sociológico, político, psicológico, histórico $)^{2}$. De allí que cuando se refiere a los componentes esenciales de este sistema se señala que son: i) los actores (organizaciones de trabajadores y empleadores y Estado); ii) las relaciones entre ellos (negociación y conflicto); y, iii) las reglas que gobiernan el sistema (estatales o pactadas). Por cierto, la ideología de los actores y el contexto en el que actúan ejercen una fuerte influencia en el sistema ${ }^{3}$.

1 Ver DUnLOP, John Thomas. Industrial Relations System. Nueva York: H. Holt and Company, 1958. Los datos consignados corresponden a la primera edición. El libro ha sido luego reimpreso en numerosas ocasiones.

2 Es importante precisar que en el Perú el término relaciones industriales ha tenido una connotación diferente, puesto que hay una profesión de Relacionista Industrial, en la que se forma a profesionales que se ocupan de los departamentos de recursos humanos, lo que, como puede apreciarse, dista mucho del sentido que le estamos dando aquí al término relaciones industriales.

3 Al respecto, ha escrito Dunlop en el prefacio del libro citado que «todo sistema de relaciones industriales crea un complejo de normas para gobernar el lugar y la comunidad de trabajo. Estas normas pueden asumir una diversidad de formas en los diferentes sistemas: convenios, estatutos,

\section{EL MODELO}

DE RELACIONES

COLECTIVAS

PERUANO: DEL IN-

TERVENCIONISTA

Y RESTRICTIVO AL

PROMOCIONAL

THE MODEL OF

PERUVIAN

COLLECTIVE

RELATIONS: FROM INTERVENTIONIST AND RESTRICTIVE TO PROMOTIONAL 
En este marco, una de las primeras discusiones cuando aterrizó esta concepción fuera del mundo anglosajón fue la denominación de esta visión científica, puesto que la traducción directa hacía referencia al mundo de la industria y el trabajo humano va más allá de la relación asalariada secundaria. Razón por la cual, y en medio de no poca polémica, hay quienes han propuesto, de manera correcta desde nuestro punto de vista, que se hable de sistema de relaciones laborales.

Finalmente, debemos señalar que dentro de este sistema no solo hay diversos enfoques teóricos (institucionalismo, pluralismo, comportamentalismo, estructuralista, radical, unitario, etcétera $)^{4}$, sino que se pueden encontrar diversos modelos que generalmente giran sobre algún elemento definitorio, cuyos enfoques no solo se plantean en díadas (como autónomomo-heterónomo, centralizadodescentralizado, anómico-articulado, etcétera), sino que pueden utilizarse simultáneamente cuando lo que se busca es la mirada más macro, es decir, la que trata de envolver los diversos componentes a partir de un gran eje. En este terreno se mueve este ensayo, tratando de dar cuenta general del modelo peruano de relaciones laborales que sigue un curso histórico de más de un siglo de protagonismo estatal restrictivo (el sindicato sigue viéndose como un sujeto bajo sospecha, al que hay que marcarle un pequeño espacio de actuación), y que se ha visto contrapuesto en la última década a dos intentos de cambiarle el signo y llevarlo a un modelo promocional, de impulso, soporte o apoyo de la autonomía colectiva.

Veamos, a continuación, los principales rasgos de un tránsito que aún no encuentra una definición clara pero que muestra diversos indicios de afianzamiento.

\section{EL MODELO INTERVENCIONISTA RESTRICTIVO}

En América Latina, con la honrosa excepción de Uruguay, el modelo presente de relaciones laborales se adscribe al denominado restrictivo (razón por la cual también se le denomina modelo tradicional latinoamericano). Las causas de la presencia de este modelo en el

órdenes, decretos, reglamentos, laudos, políticas, prácticas y costumbres. La forma de la norma no altera su carácter esencial: el de definir el estatus de los actores en el lugar y comunidad de trabajo. Se considera a los actores de un sistema de relaciones industriales como permanentemente enfrentados a un contexto ambiental. El medio ambiente se compone de tres contextos interrelacionados: la tecnología, las restricciones del mercado o presupuestarias, y las relaciones de poder y estatus entre los actores. El sistema se encuentra ligado mediante una ideología o por concepciones compartidas por todos los actores. La tarea central de una teoría de las relaciones industriales es la de explicar por qué se establecen determinadas normas en determinados sistemas de relaciones industriales y cómo y por qué cambian en respuesta a los cambios que afectan el sistema» (DUNLOP, John Thomas. Industrial Relations System. Londres/Ámsterdam: Feffer and Simons, p. viii).

4 Sobre el particular, ver OJEDA AviLÉS, Antonio. Derecho sindical. Octava edición. Madrid: Tecnos, 2003, pp. 88 y ss.; y el ensayo de CEDROLA SPREMOLLA, Gerardo. «Los enfoques teóricos en relaciones laborales». Relasur, 2 (1994), pp. 49-74. 
continente americano y en nuestro país son varias, pero, en términos generales, Ermida Uriarte identifica los siguientes factores ${ }^{5}$ :

a. Causas económicas: el desarrollo económico de Latinoamérica fue tardío en comparación con las naciones potencias del orbe, siendo aún una economía preponderantemente productora de materia prima, con la presencia de un sector industrial pequeño y atrasado tecnológicamente, la existencia de un sector público considerablemente grande, y la emergencia, cada vez con mayor fuerza, de un pujante sector informal (características del denominado fordismo periférico), lo que retrasó el desarrollo industrial del que ya gozaban los países europeos. Por lo expuesto, la industria en nuestro continente se mantuvo atomizada y sin extenderse a todos los sectores de producción.

La repercusión sobre el hecho sindical es evidente, ya que las organizaciones sindicales creadas estaban limitadas al ámbito de la empresa, no pudiendo generar una negociación de nivel mayor, ni provocar ni administrar un conflicto de ámbito más amplio. Por consiguiente, los sindicatos creados eran incapaces de generar «un derecho laboral autónomo con vocación de generalidad; a lo más, podrían generar algunas normas autónomas muy descentralizadas y concretas» ${ }^{6}$. La respuesta del Estado ante la falta de normas autónomas de ámbito nacional fue la creación de normas estáticas, abstractas y de aplicación general.

b. Causas históricas culturales: el derecho latinoamericano, en todas sus ramas, nació como un sistema jurídico cerrado y escrito que tiende a identificar derecho con ley, producto de la influencia recibida por el derecho romano, español, portugués y francés, todos ellos sistemas burocráticos, administrativistas y estatistas. En ese sentido, el producto normativo de la negociación colectiva —el convenio colectivo- no encaja en la concepción reduccionista propia del derecho latinoamericano.

Por cierto, no hay que dejar de lado el factor político, pues el Estado latinoamericano ha mostrado en términos generales una obsesión por el control político del sindicato y de su acción, a través de su reglamentación exhaustiva y la falta de tratamiento normativo de contenidos promotores de la acción sindical.

c. Causas sindicales: Los factores antes mencionados han producido un movimiento sindical débil en esta parte del continente, el mismo que retroalimenta el reglamentarismo e intervencionismo estatal. En efecto, el fenómeno sindical latinoamericano carece de

5 Ver ERMIDA URIARTE, Óscar. «La intervención estatal en las relaciones colectivas de trabajo latinoamericanas». Revista Nueva Sociedad, 128 (1993), pp. 29-34.

6 Ibídem, p. 30.

EL MODELO DE RELACIONES COLECTIVAS

PERUANO: DEL INTERVENCIONISTA Y RESTRICTIVO AL PROMOCIONAL

THE MODEL OF

PERUVIAN

COLLECTIVE

RELATIONS: FROM INTERVENTIONIST AND RESTRICTIVE TO PROMOTIONAL 
fuerza para plantear oposición a las imposiciones gubernamentales y del empleador, generándose un círculo vicioso.

Sobre la base de estas causas, el modelo latinoamericano, con la honrosa excepción de Uruguay, se ha impuesto, generalmente desde el Poder Ejecutivo, con regulaciones detalladísimas, dadas bajo la convicción de que el conflicto laboral tiene como causa la existencia de los sindicatos y que, por tanto, hay que tratar de controlar al máximo su surgimiento, configuración y actuación, para garantizar la paz social.

Sobre estas bases, veamos en detalle las características del modelo peruano, muy determinado por un rol hiperdesarrollado del Estado, a partir fundamentalmente de lo regulado por el decreto legislativo 25593 (en adelante, LRCT), cuyo texto único ordenado fue aprobado por el decreto supremo 010-2003-TR.

Nos encontramos con una regulación heterónoma y que no fue negociada con los actores del sistema de relaciones laborales. Si bien en el Poder Legislativo había un importante número de proyectos de ley respecto del tema, esta norma se aprobó cuando el Parlamento estaba cerrado por el golpe de Estado, sin ninguna discusión ni política ni menos de consulta a las organizaciones sindicales y empresariales. Existen experiencias, como la española (el título III del Estatuto de los Trabajadores que disciplina el derecho de negociación colectiva), en la que las regulaciones en materia de relaciones colectivas no solo fueron discutidas con los sujetos colectivos, sino que fueron objeto de acuerdo y, por tanto, de legislación negociada, con la finalidad de garantizarles el máximo nivel de cumplimiento por su carácter consensual. Pero, evidentemente, este no fue el caso peruano, sino que más bien se dio el caso contrario, en el que el Poder Ejecutivo ha reclamado para sí el monopolio de la regulación de las relaciones colectivas.

Se trata de una regulación que se desmarca de la tendencia latinoamericana hacia el impulso de la autonomía colectiva (Uruguay, Brasil, Venezuela, República Dominicana y Paraguay) y que, más bien, como ya se mencionara, es una de las expresiones más acabadas del modelo tradicional latinoamericano de regulación de las relaciones colectivas de trabajo, ratificando sus rasgos autoritario-reglamentarista, controlista y restrictivo. El Estado regula prácticamente todo lo concerniente al surgimiento, configuración y actuación del sujeto colectivo, dejando a la autonomía sindical y a la autonomía colectiva un espacio verdaderamente estrecho y limitado. Y ello puede constatarse históricamente, puesto que el único campo de las relaciones laborales peruanas que no ha sido objeto de reforma es el de las relaciones colectivas, en donde sigue primando el espíritu que insufló vida a las primeras normas de comienzos del siglo XX. Todo ello, en abierta contradicción con las normas constitucionales que desde 1979 
ordenaban una intervención de signo opuesto: garantistas y de fomento de la actuación colectiva (aquella óptica invertida del legislador que denunciara Sanguineti en 1994)?

En función de ello, es una regulación totalizadora y prácticamente excluyente de la regulación estatal de las relaciones colectivas, que define un modelo carente de toda autonomía externa (frente a las reglas estatales), con lo que, en consecuencia, se impide que sean las propias partes sociales, normalmente a nivel de sus representaciones de cúpula, las que puedan ir creando autónomamente un ordenamiento intersindical como el existente en los países con un sistema de relaciones laborales maduro, en el que, con la negociación colectiva como instrumento de gobierno de este ordenamiento, se pactan todas las reglas necesarias para la expresión de su poder normativo y para resolver todos los conflictos de creación, aplicación o interpretación que surjan a su alrededor. $\mathrm{Al}$ respecto, no se debe olvidar la estrechísima relación inversamente proporcional entre el papel del Estado y los márgenes de actuación de la autonomía colectiva, que en este caso importan un rol estatal tan sofocante cuya consecuencia es dejar sin el mínimo espacio vital a la autonomía colectiva.

Es una regulación que por primera vez trató sistemáticamente los tres institutos principales que conforman la autonomía colectiva, por lo que resulta más coherente que la regulación precedente. En tal sentido, la LRCT supera la dispersión normativa existente sobre el particular, que llevaba a que convivan un decreto supremo de 1913 (del 28 de enero, que regulaba la huelga), otro decreto supremo de 1961 (número 011, que regulaba la organización de sindicatos) y otro decreto supremo (006-71-TR, que regulaba la negociación colectiva). La coherencia de la regulación de los tres institutos no solo implica una disciplina más homogénea, sino que se expresa en la misma visión restrictiva y controlista que la informa.

Se establece un modelo descentralizado de relaciones laborales, en el que lo sindical gira alrededor de la empresa. Se favorece abiertamente la sindicación en este ámbito, lo mismo que la negociación colectiva y la huelga, concentrando la conflictividad laboral en la sede empresarial. Además de ello, la tercera disposición transitoria y final de la LRCT marcó el fin de las pocas negociaciones colectivas de rama de actividad, al obligar a ratificar o modificar el nivel sectorial, estableciendo que a falta de acuerdo al respecto la negociación colectiva debería llevarse a cabo a nivel empresarial. Asimismo, el artículo 46 de

7 SANGUINETI RAYMOND, Wilfredo, «Los sindicatos y la libertad sindical en la nueva Ley de Relaciones Colectivas de Trabajo (Decreto Ley 25593)". En AUTORES VARIOs. Quo vadis jus? Estudios jurídicos en homenaje al Profesor Ricardo La Hoz Tirado. Lima: Universidad Nacional Mayor de San Marcos/ Facultad de Derecho y Ciencias Políticas, 1994, pp. 258-295.

EL MODELO DE RELACIONES COLECTIVAS

PERUANO: DEL INTERVENCIONISTA Y RESTRICTIVO AL PROMOCIONAL

THE MODEL OF

PERUVIAN

COLLECTIVE

RELATIONS: FROM INTERVENTIONIST AND RESTRICTIVE TO PROMOTIONAL 
la LRCT se encargó de impedir cualquier intento futuro de negociación colectiva a este nivel, al colocar requisitos de representación mayoritaria de trabajadores y empresas de imposible cumplimiento en un país donde la micro y pequeña empresa (en la que no cabe el sindicato de empresa) significan tres cuartas partes del total. Dada la interdependencia de las facultades que integran la autonomía colectiva, sin lugar a dudas que la imposición en la práctica de la negociación colectiva a nivel de empresa condujo a una organización y conflictividad también en ese ámbito.

A nivel de creación y configuración de organizaciones sindicales, se busca predefinir al sujeto social, restringir al máximo a sus titulares, establecer una estructura sindical cerrada (cuatro tipos de sindicatos), imponerle fines y prohibirle ámbitos de actuación, concederle límites a su actuación a través de una disfuncional duplicidad de personalidades jurídicas. Se impone también un registro manejado por el Poder Ejecutivo y que puede ser cancelado por el registrador sin ninguna intervención judicial, etcétera. A ello habría que agregarle que en un país de estructura sindical empresarial en el que, además, se negocia casi únicamente en ese nivel (salvo construcción civil y estibadores portuarios), se impone un número mínimo de veinte afiliados para constituir un sindicato, dejando al 75\% de la PEA asalariada sin posibilidades reales de ejercer su libertad sindical.

En lo que hace al funcionamiento del sistema de relaciones colectivas de trabajo, se lo asimila al modelo estático que hace girar la actividad sindical principalmente alrededor de la negociación colectiva, con lo que tras arribar al convenio colectivo, la representación sindical se reduce a mínimos, sin que exista siquiera comisiones paritarias que continúen funcionando para resolver los problemas de aplicación o interpretación del acuerdo. Además de ello, se impone una estructura negocial descentralizada, al establecer que el nivel de negociación se define por acuerdo de partes y que, a falta de este, se negociará a nivel de empresa (lo que vuelve inútiles a los sindicatos supraempresariales), con lo que se opta por la posibilidad de menor cobertura negocial, contraviniendo el deber constitucional de fomento de la negociación colectiva (artículo 28.2). No se regula en detalle el deber de negociar de buena fe, acogiendo únicamente una regulación defectuosa e incompleta del deber de información, puesto que no se establece el plazo en que el empleador debe entregar la información, y en muchos casos se entrega mucho después de elaborado y presentado el pliego de peticiones, por ejemplo.

Se establece un modelo mixto en lo que se refiere a la titularidad del interés colectivo, ya que, en el terreno negocial, se la otorga a la organización sindical y solo en ausencia de ella a delegados elegidos para tal fin (modelo orgánico, cuyo paradigma es Alemania), en tanto que en materia de huelga se diseña una titularidad de carácter individual con un ejercicio colectivo (modelo individualista, cuyo paradigma es 
Francia, aunque con un protagonismo sindical evidente en su sistema de relaciones laborales), dejando al sindicato en un rol subordinado, que con las reformas originadas por el Tratado de Libre Comercio (TLC) con EEUU están permitiendo un rol mayor a la organización de los trabajadores. Esta ausencia de un paradigma único tiene como consecuencia inevitable la ausencia de un criterio que resuelva el problema de comunicación o incomunicación entre la negociación colectiva y la huelga, en la medida en que al ser titulares distintos no cabría, por ejemplo, deducir la existencia de un deber de paz inmanente tras la firma de un convenio colectivo, ya que el derecho de huelga seguiría siendo un derecho fundamental de los trabajadores que no podría enervarse por un pacto del sindicato.

El tratamiento del conflicto obedece a una consideración poco menos que patológica del mismo, de modo que se le brinda un espacio mínimo de expresión, configurando una regulación que se adscribe a modelo casi contractual, al permitirse la huelga fundamentalmente dentro del proceso de negociación colectiva, con requisitos muy exigentes (mayoría absoluta de los trabajadores de la empresa o unidad ${ }^{8}$ ) e ilegalizando todas las modalidades de huelga que no consistan en la suspensión total de actividades, con abandono del centro de trabajo y para fines estrictamente profesionales. Se prevé una posibilidad de huelga frente a incumplimientos legales o convencionales del empleador, pero solo una vez que se ha obtenido una sentencia judicial firme al respecto, lo que impide la materialización práctica de la medida de presión.

En consonancia con todo lo dicho, la administración laboral, salvo honrosísimas excepciones ${ }^{9}$, no solo ha aplicado las normas legales con especial esmero y diligencia, sino que ha aprobado reglamentos y directivas que han profundizado los rasgos antes anotados, ha emitido resoluciones para los casos concretos que han hecho muy difícil la obtención del registro sindical, imposible la negociación colectiva supraempresarial o ilusoria la declaratoria de legalidad de una huelga por más que cumpla con todos los requisitos legales.

Por lo expuesto, en la apreciación general que se puede extraer de esta regulación resalta la valoración negativa que tiene el legislador del fenómeno sindical que, más allá de contrastar con su carácter de patrimonio jurídico de la humanidad y su reconocimiento constitucional, nos muestra el carácter autoritario y excluyente que lo informa, lo cual resulta totalmente reprochable jurídicamente como veremos a continuación.

8 La exigencia de mayorías también se ha relativizado como consecuencia de las exigencias norteamericanas originadas en el TLC.

9 Entre julio de 1985 y julio de 1986, en primer lugar; entre marzo de 2004 y marzo de 2005; y, en cierta medida, desde julio de 2011.

EL MODELO

DE RELACIONES

COLECTIVAS

PERUANO: DEL IN-

TERVENCIONISTA

Y RESTRICTIVO AL

PROMOCIONAL

THE MODEL OF

PERUVIAN

COLLECTIVE

RELATIONS: FROM INTERVENTIONIST AND RESTRICTIVE TO PROMOTIONAL 


\section{CUESTIONAMIENTOS JURÍDICOS Y ECONÓMICOS}

AL MODELO INTERVENCIONISTA EN EL PERÚ El modelo intervencionista, como destaca $\mathrm{Neves}^{10}$, se ve cuestionado en el Perú a partir de fines de los setenta, tanto en el plano jurídico, por la instauración de un nuevo marco constitucional, cuanto en el plano económico, por la crisis del fordismo y la irrupción de la flexibilidad laboral.

En el plano jurídico, en 1979 se había aprobado una nueva Constitución, que entraría en vigencia plena en 1980 con el retorno a la democracia. Esta Constitución se adscribía explícitamente al modelo de Estado Social de Derecho, lo que en el campo laboral se expresaba en la consagración de un modelo democrático de relaciones laborales (que explicaremos más adelante), a partir del reconocimiento pleno, expreso y amplio de la autonomía colectiva y de la imposición al Estado de una intervención de fomento y garantía de los derechos y facultades que la integran. Además, otorgaba jerarquía constitucional a los Tratados sobre Derechos Humanos Fundamentales, lo que tenía una especial trascendencia respecto de los Convenios Internacionales de Trabajo de la Organización Internacional del Trabajo (OIT).

Este modelo era incompatible con la legislación preconstitucional intervencionista y restrictiva, ya que importaba un conjunto de obligaciones para todo el Estado, dirigidas a promover y garantizar la eficacia de los derechos colectivos y dar las facilidades para que cumplan sus funciones, partiendo de la básica: regular las condiciones de trabajo.

Esta constitucionalización de la autonomía colectiva no es gratuita: se basa en la idoneidad que tiene el fenómeno de la actuación organizada de los trabajadores para equilibrar la desigual relación existente entre las partes de la relación individual del trabajo; para canalizar y componer el conflicto inherente a tal relación de manera que pueda rendir sus mejores frutos en materia de igualdad y de justicia social, funcionalizándolo respecto de tan importantes objetivos del Estado; para producir normas adecuadas y consensuadas respecto de cada una de las múltiples unidades productivas que permitan un máximo de eficiencia económica; para servir de instrumento de garantía de la aplicación del Derecho del Trabajo en su conjunto, habida cuenta de su bajísima tasa de aplicación ${ }^{11}$; para asegurar un grado razonable de participación de los trabajadores en la defensa de sus intereses a nivel

10 NeVES MUJICA, Javier. «Balance de la Reforma Laboral ». Revista Asesoría Laboral. Lima, diciembre 2000 , p. 8.

11 En tal sentido, señala Kahn-Freund que «en lo que respecta a las relaciones laborales, las normas legales carecen a menudo de eficacia, si no se encuentran además reforzadas por sanciones sociales, es decir, por el poder equilibrador de los sindicatos y demás organizaciones de trabajadores expresado a través de la consulta y negociación con el empresario, y, en último término, si esta falta, mediante el cese concertado de trabajo», concluyendo en el mismo texto que "las leyes tratan de limitar el poder de mando del empresario. Su éxito en este punto depende del grado de organización 
general; y, finalmente, para cumplir el rol de cohesión e integración social (en síntesis, democracia material) que permita superar nuestra secular sociedad excluyente y sustituirla por otra de la que todos nos sintamos parte aunque sea mínimamente.

Por todas estas razones es que la autonomía colectiva, a pesar de las turbulencias que ha traído consigo el último cuarto de siglo, goza de buena salud y mucho predicamento en los países más democráticos del mundo y ha sido considerada como patrimonio jurídico de la humanidad, a partir del reconocimiento universal de la libertad sindical, que viene a ser la condensación jurídica de la autonomía colectiva. En función de ello habría que entender en su plenitud aquellas expresiones de grandes maestros del Derecho del Trabajo contemporáneo como Verdier, Giugni o Lyon-Caen, cuando plantean que «la libertad sindical es un denominador común de los regímenes democráticos», que «la efectividad del orden democrático puede ser medida por la eficacia de la libertad sindical», o que "no hay democracia sin libertad sindical», respectivamente ${ }^{12}$.

La Constitución de 1993, siendo bastante más lacónica que la de 1979, consagró también la autonomía colectiva en sus tres principales manifestaciones y ordenó, nuevamente, al Estado garantizarla y fomentarla, con lo que las reflexiones anteriores son plenamente válidas también para la norma suprema vigente.

Esta refundación jurídica que trajo consigo la Constitución de 1979, y que se mantiene en la Carta Magna actual, exigía un replanteamiento total de la regulación infraconstitucional de corte profundamente limitadora y reglamentarista. De allí que jurídicamente se hacía insoslayable una profunda reforma de la regulación de las relaciones colectivas de trabajo, que tuviese como finalidad fomentar y garantizar el surgimiento y la actuación eficaz de los sujetos colectivos, generándoles un espacio de encuentro en el que, a la par que reconocían sus intereses contrapuestos y comunes, la conjugación de estos los llevaba a sentirse parte de un proyecto común de democracia industrial y social, es decir, de un país con un tejido social consolidado y complejo en el que cada grupo social y cada integrante de ellos tenía un papel y un reconocimiento.

En el plano económico también se planteaban exigencias de reforma: la crisis del fordismo y la difusión de las nuevas tecnologías que dieron lugar al modelo toyotista y sus bases de descentralización productiva y especialización flexible, significaron la crisis de las regulaciones generales y rígidas y pusieron en el primer plano la necesidad de regulaciones

de los trabajadores» (KAHN-FREUND, Otto. Trabajo y Derecho. Traducción de Jesús Galiana. Madrid: Ministerio de Trabajo y Seguridad Social, 1987, p. 55).

12 Ver ERMIDA URIARTE, Oscar \& Alfredo VILLAVICENCIO Ríos. Sindicatos en libertad sindical. Lima: ADEC-ATC, 1991, p. 28. 
diversificadas y adaptables, versátiles y flexibles, coherentes con el incremento de productividad necesario para ser competitivos.

Aparece en el escenario así, como prima donna, la flexibilidad laboral. Ahora bien, si se revisan todas las definiciones serias de este sistema, se verá que el elemento común es la adaptabilidad, la versatilidad para adecuarse rápidamente a nuevos contextos. Y en este terreno, si algo caracteriza al Derecho del Trabajo frente a las otras ramas del ordenamiento jurídico es que tiene una fuente normativa consensual y de múltiples sedes, con muchas ventajas claras frente a la otra fuente (la legal): el convenio colectivo.

En tal sentido, esta fuente reguladora se ha convertido en «el método predominante para la fijación de normas laborales $»^{13} \mathrm{o}$ «sin lugar a dudas, el mecanismo predominante, el mecanismo principal de regulación de las relaciones laborales» ${ }^{14}$, porque su origen consensual, el conocimiento detallado de la realidad a normar, su duración limitada en el tiempo, le dan unas idoneidad, equilibrio y aceptación inconseguibles desde la heterorregulación estatal. La situación de cada unidad productiva es peculiar, por tanto, las normas que regulen sus relaciones laborales deben tener un grado de especificidad y flexibilidad muy grande, y ello solo es posible desde la autonomía colectiva.

Esta adecuación de la norma colectiva, unida a su origen consensual, le da un índice de aceptación y cumplimiento mucho mayor que el de las normas impuestas desde el Estado. De allí que exista también un criterio de eficiencia económica que lleva a apuntalar la regulación colectiva de las relaciones laborales, frente a la lejanía del objeto a regular, la mayor rigidez e imposición que significaría otorgarle un papel protagónico al Estado en este terreno y frente a la desprotección total de los trabajadores que supondría dejarlos a merced del establecimiento individual de las condiciones de trabajo. Por ello, nuevamente, su rol tiene que dirigirse a fomentar el surgimiento y la actuación eficaz del sindicato para que la autonomía colectiva pueda establecer las reglas eficientes, apropiadas y justas en materia laboral.

Había, por tanto, razones jurídicas y económicas que planteaban perentoriamente la necesidad de una reforma legislativa de las relaciones colectivas de trabajo y las dos apuntaban en la misma dirección: favorecer el surgimiento de las organizaciones sindicales y fortalecer su actuación, utilizando el camino de la negociación colectiva para la adecuación del estatuto normativo de cada unidad productiva a sus cambiantes posibilidades y contextos.

13 WindmULleR, John Philip. «Nueva consideración de la negociación colectiva en los países industrializados». Informe para la Organización Internacional del Trabajo, Ministerio de Trabajo y Seguridad Social. Madrid: 1987, p. 20.

14 OIT. Informe de la Comisión de Expertos en la Aplicación de Convenios y Recomendaciones, Informe III (Parte 1.A). Ginebra: 1999, p. 5. 
En tal sentido, contrariamente a las exigencias jurídicas y económicas ya vistas, a las que se debería agregar una mayor necesidad de fortalecimiento de las organizaciones colectivas para compensar la retirada estatal del ámbito individual, con el fin de mantener el equilibrio necesario para el mejor funcionamiento productivo y social de las relaciones laborales, se buscó favorecer la posición empresarial para conseguir la generación de mayor y mejor empleo, lo que, por supuesto, no ha sucedido, siendo el sacrificio en los niveles de protección del trabajador y la casi desaparición de los sujetos colectivos las consecuencias directas de la normativa laboral aprobada en los años noventa, que ahora deben revertirse en la búsqueda de un nuevo paradigma laboral que restaure el equilibrio en las relaciones de trabajo y apuntale un sistema de relaciones laborales también socialmente eficiente.

\section{EL MODELO DEMOCRÁTICO}

Frente al modelo histórico intervencionista analizado, se ha buscado sentar las bases de un sistema democrático de relaciones laborales basado en el impulso de sujetos institucionalizados, vitales y sólidos, que ordenen las relaciones laborales de la manera más adecuada para poder afrontar los retos del nuevo paradigma productivo que ha venido de la mano de la globalización, generando las regulaciones adaptables, versátiles y coherentes con un modelo de incremento permanente de productividad, sin descuidar los equilibrios básicos de todo Estado Social de Derecho.

Este modelo implica la presencia gravitante de la autonomía colectiva como la institución en la que se expresa el papel preponderante que deben tener las representaciones de trabajadores y de empleadores para autorregular sus intereses contrapuestos. Se trata, a fin de cuentas, de un sistema de autonomía máxima y heteronomía mínima (vinculada fundamentalmente al campo de los intereses generales o públicos). Los rasgos principales que presenta este modelo son ${ }^{15}$ :

a. Concepción dialéctica de las relaciones de trabajo y asignación al conflicto de un papel funcional dentro del esquema constitucional. Se parte del principio fundamental de que trabajadores y empleadores son portadores de intereses diferenciados que se encuentran en contraposición y conflicto, y que precisamente el ordenamiento jurídico-laboral se propone integrar. Para esto, se definen los sujetos colectivos necesarios para la representación y defensa de los intereses contrapuestos en juego y, además, se definen los derechos de conflicto básicos. 
b. Consagración de la autonomía colectiva como pieza esencial del sistema normativo. Esto hace que el papel del Estado como fuente exclusiva o preferente de las relaciones laborales se vea sustituido por el de los actores sociales.

c. Intervención promocional y alimentadora del sistema por parte del Estado como noción de cierre de excepcional trascendencia. Reiteramos que no se postula un sistema de abstention of law, sino uno en el que el Estado juega un rol fundamental: apuntalar la mayor igualdad posible de fuerzas entre las representaciones colectivas, para que luego estas puedan hacerse cargo de la regulación de las relaciones laborales en su conjunto. Se trata de cambiar la intervención estatal y otorgarle no solo un rol de eliminación de obstáculos respecto de los sujetos colectivos y de su actuación, sino también el rol de generar los canales para garantizar el ejercicio de estos derechos y para otorgar las facilidades a fin de que desplieguen su máxima eficacia.

A partir de lo escuetamente dicho, creemos firmemente que este modelo debe procurar una refundación de la relación entre el Estado, los empleadores y las organizaciones de trabajadores, cuyo núcleo central debe asentarse en el reconocimiento de las altísimas funciones que cumple la actuación colectiva de los trabajadores en una sociedad democrática, en los siguientes y trascendentales terrenos:

a. Equilibrio de fuerzas con la parte de los empleadores y la consiguiente consecución de niveles cada vez mayores de igualdad sustancial, participación y justicia social (democracia industrial).

b. Composición y funcionalización del conflicto industrial, acabando con la visión patológica del mismo y generándole los canales más apropiados para que se potencien sus consecuencias positivas y se limiten las negativas.

c. Producción de normas idóneas para cada unidad productiva a través de convenios colectivos que son pactados por quienes conocen mejor que nadie la situación de cada una de ellas.

d. Vigencia real del Derecho del Trabajo a partir de la acción vigilante de los sindicatos, permitiendo mejorar la baja tasa de aplicación que genéticamente tiene esta disciplina jurídica.

e. Representación general de los intereses de los trabajadores en la sociedad y ante el Estado, en la medida en que los sindicatos no solo actúan como agentes económicos a través de la negociación colectiva, sino que también lo hacen como agentes sociales que expresan la identidad social de los trabajadores, frente a los demás 
actores sociales y frente al Estado, generando así un interrelación muy estrecha entre democracia y libertad sindical.

f. Conquista cotidiana de grados cada vez mayores de cohesión social, sobre los cuales se ha de empezar a superar el carácter excluyente de nuestra sociedad y se ha de edificar otra de la que todos seamos parte y de la cual nos sintamos parte.

En el terreno laboral, un sistema democrático de relaciones laborales implica, pues, la presencia gravitante de la autonomía colectiva como la institución en la que se expresa el papel preponderante que deben tener las representaciones de trabajadores y de empleadores para autorregular sus intereses contrapuestos. Se trata de un sistema de autonomía máxima y de heteronomía mínima, como ya señalamos.

Junto con tal redefinición de la filosofía de la norma (que conlleva una significativa ampliación de la autonomía externa), el modelo democrático de relaciones laborales que se describe debe superar la ancestral atomización de la actuación colectiva y dar pasos significativos hacia su centralización, a partir del fomento de la negociación colectiva sectorial, en la medida en que, contando con una estructura empresarial atomizada en nuestro país, lo único racional es la organización y actuación colectiva a nivel sectorial, tanto para que la cobertura (eficacia) sea significativa, cuanto para establecer cargas comunes que eviten las tentaciones de hacer reposar la competencia empresarial en el dumping social (la reducción de las remuneraciones y condiciones de trabajo). Asimismo, para enfrentar las limitaciones de la negociación sectorial sería conveniente facilitar la articulación de la negociación colectiva (reducir la autonomía interna del modelo), de manera que se facilite que, tras las pautas generales previstas en la negociación sectorial, a nivel empresarial se puedan establecer precisiones, adecuaciones o desarrollos acordes con la realidad de cada unidad productiva.

Finalmente, este rol garantista tiene que actuar en los tres componentes centrales en que se expresa la autonomía colectiva, habida cuenta de su interdependencia: en el surgimiento de los sujetos colectivos (sindicación o auto organización, como presupuesto), en el de la producción de normas pactadas (negociación colectiva o autorregulación, como expresión del poder normativo del que está investida) y en el de las medidas de presión (huelga o autotutela, como garantía de eficacia). De no ser así, por limitarse excesivamente alguno o algunos de estos componentes, el modelo se vacía totalmente de contenido y no pasa de tener una simple apariencia democrática.

\section{EL MODELO DE RELACIONES COLECTIVAS \\ PERUANO: DEL IN- TERVENCIONISTA Y RESTRICTIVO AL PROMOCIONAL}

THE MODEL OF

PERUVIAN

COLLECTIVE

RELATIONS: FROM INTERVENTIONIST AND RESTRICTIVE TO PROMOTIONAL 


\section{EL MODELO PROMOCIONAL DE RELACIONES LABORALES EN EL PROYECTO DE LEY GENERAL DEL TRABAJO}

Partiendo del mandato constitucional de garantía de la libertad sindical y fomento de la negociación colectiva (artículo 28.1 y 2), que implica una radical redefinición de la filosofía que inspira cualquier regulación sobre relaciones colectivas, en el año 2002, la Comisión de Trabajo del Congreso de la República creo una Comisión de Expertos a la que se le encargó la elaboración de una propuesta de Ley General del Trabajo que sistematizara toda la legislación vigente y regulase tanto las relaciones individuales y colectivas, devolviendo el equilibrio a las partes sociales. En julio de tal año la Comisión entregó el Anteproyecto encomendado y desde entonces, hasta el año 2007, se discutió esta iniciativa en el seno del Consejo Nacional del Trabajo. En esta institución las partes sociales llegaron a consensuar hasta casi el 90\% de un texto que luego el Ministerio de Trabajo y Promoción del Empleo presentaría al Congreso.

El 1 de setiembre de 2011, el Ministerio de Trabajo y Promoción del Empleo, mediante la resolución ministerial 257-2011 constituyó una Comisión de Expertos encargada de revisar y actualizar el proyecto de la Ley General de Trabajo, que entregó su propuesta normativa en diciembre de tal año. Veamos las características centrales que informan al modelo propuesto por la Comisión de Expertos.

En primer lugar, el modelo se enmarca dentro del nuevo contexto latinoamericano de impulso de la autonomía colectiva que se vive en países tales como Brasil, Paraguay, República Dominicana, Uruguay, entre otros. En segundo lugar, se trata de una propuesta que viene refrendada por un elevadísimo consenso social, puesto que la Comisión de Expertos ha respetado casi la totalidad de los consensos sociales existentes en el Consejo Nacional del Trabajo. En tercer lugar, y de manera adicional a esta legitimidad social, se busca que tenga también legitimidad política, en la medida en que se busca que sea una ley aprobada por el parlamento, con la pluralidad que ello importa, lo que por sí solo resulta resaltable porque ello nunca ha sucedido a lo largo de nuestra historia.

En cuarto lugar, estamos ante una propuesta de regulación bastante «oitiana», en la medida en que muestra un respeto muy grande a las normas internacionales y su desarrollo e interpretación a través de los pronunciamientos del Comité de Libertad Sindical y de la Comisión de Expertos en la Aplicación de Convenios y Recomendaciones. Ello, a su vez, nos hace ver que, acorde con el carácter mínimo de las normas internacionales, se está en un primer peldaño normativo, lo que va a tono con el estadio de refundación y con lo posible en la coyuntura actual. 
Además de ello, se propone un modelo que todavía tiene una autonomía externa limitada, es decir, el papel de la regulación estatal es muy fuerte en el ámbito de la autonomía colectiva, lo que responde a nuestras coordenadas históricas, aunque se han abierto todas las puertas para que sean las partes sociales las que vayan reclamando para sí la regulación no solo sustantiva sino también estructural de las relaciones laborales.

En sexto lugar, en el ámbito de la autonomía interna se busca generar las condiciones para que el sistema pueda contar con una negociación colectiva articulada que nos permita aprovechar los beneficios de la negociación sectorial y de la negociación empresarial relativizando los lados menos favorables que tiene cada una de estas negociaciones.

En séptimo lugar, es un modelo que impulsa la paulatina sustitución del modelo descentralizado y disfuncional para el funcionamiento del país, estableciendo una cierta preferencia por la negociación sectorial que le dé racionalidad económica, amplíe su cobertura y evite el dumping social como instrumento de competencia entre empresas.

Finalmente, la propuesta de Ley General del Trabajo reseñada aborda el conflicto sin hipocresía, sin considerarlo patológico sotto voce, sino como inherente a las relaciones laborales, por lo que busca que sus expresiones no sean negadas a priori sino que sean procesadas a través de instrumentos de composición que le permitan jugar el rol dinámico y de tutela de los intereses en juego en los mejores términos, es decir, en los términos que los propios actores de las relaciones laborales consideren.

A continuación, vemos cómo se concretan estas líneas maestras en la regulación específica de la sindicación, negociación colectiva y huelga, desde el prisma que consideramos neurálgico: el fomento o promoción de la autonomía colectiva.

El proyecto de la Ley General del Trabajo busca establecer un modelo democrático de relaciones laborales en el Perú, porque, en consonancia con las características centrales de este modelo, se centra en la promoción o fomento de la negociación colectiva, es decir, busca generar las condiciones para que sean las representaciones empresariales y sindicales quienes regulen las condiciones de trabajo y las relaciones laborales tanto estructural como sustancialmente.

Ello no solo porque de facto en el país la tutela colectiva se ha pulverizado en los últimos veinte años, pasando, por ejemplo, de 2500 a 500 convenios colectivos, desde fines de los años ochenta, con gran responsabilidad estatal (legal y administrativa ) en esta debacle; sino porque hay que apoyar la construcción de un Estado Constitucional de Derecho, comenzando por cumplir, por primera vez en nuestra historia, con el prístino mandato constitucional de fomento de la negociación colectiva previsto por el artículo 28.2 de la norma suprema. 
Para tal fin, podemos dividir el proyecto en tres grandes bloques promocionales: genérico, específico y colateral. En el primer caso, de entrada, la LGT trae dos disposiciones que marcan la impronta promotora que tiñe su regulación y debe teñir su interpretación y aplicación. La primera de ellas es el artículo XXIV del Título Preliminar, en donde se establece, con claridad que nos releva de mayor explicación, que

«el Estado orienta y sujeta su actuación al cumplimiento y garantía de los derechos reconocidos en la Constitución Política, los Tratados Internacionales ratificados por el Perú, las demás normas laborales y los criterios establecidos por los tribunales internacionales a los que el Perú está sujeto, el Tribunal Constitucional y la Corte Suprema de Justicia. En materia de interpretación normativa se consideran los pronunciamientos de los órganos de control de la Organización Internacional del Trabajo OIT», el que debe leerse en directa concordancia con el artículo XV que dispone que «los tratados sobre derechos humanos, en especial los Convenios Internacionales del Trabajo de la Organización Internacional del Trabajo ratificados por el Perú, integran el bloque de constitucionalidad de los derechos fundamentales laborales enumerados en la Constitución Política del Perú».

Con estas disposiciones se deja muy claramente establecido que el Estado debe tener un rol muy activo en la defensa y garantía de los derechos fundamentales, entre los que se encuentra la libertad sindical (y por ende la negociación colectiva), sujetando su actuación a los mandatos constitucionales, tanto los originarios como los derivados de los convenios de la OIT (bloque de constitucionalidad), y considerando además en materia interpretativa los pronunciamientos del Comité de Libertad Sindical y de la Comisión de Expertos en Aplicación de Convenios y Recomendaciones de la OIT, que son imprescindibles en la precisión del contenido concreto de los convenios 87, 98, 135, 151 y 154, para mencionar a los más relevantes en este campo.

La segunda referencia genérica es el artículo 346 que busca comprometer al Estado en el establecimiento de estímulos económicos concretos para los beneficios que se otorguen vía negociación colectiva, tales como tratamiento tributario más ventajoso u otros incentivos específicos, de modo que los empleadores encuentren un retorno económico puro y duro por pactar colectivamente con los representantes de los trabajadores.

En lo que hace al bloque específico de fomento, en la LGT se ha partido de entender la negociación colectiva como parte inescindible del complejo mecanismo constitucional de tutela colectiva, cuyo presupuesto es la sindicación y cuyas expresiones estrictas son la negociación y el convenio colectivo y cuya garantía de eficacia es la huelga. 
Por tanto, para promoverla o impulsarla se han eliminado, por ejemplo, las normas restrictivas actuales en lo que hace a fines, requisitos y procedimientos vinculados a trabajadores y organizaciones sindicales y se han adoptado disposiciones que les permitan a ambos adecuarse a los tiempos de descentralización productiva y dispersión del colectivo laboral, reconociéndoles su derecho a formar sindicatos de grupo de empresa, de contratas y subcontratas, conjuntos (empresa principal y contratas), y todos los demás que ellos consideren idóneo para defender sus intereses. A la par, se ha atacado directamente el principal escollo para la vigencia de la libertad sindical en las dos últimas décadas: la ausencia de tutela efectiva para quien constituye o se afilia a un sindicato, estableciendo, en primer lugar, una tutela general que anula y priva de efectos a todo precepto normativo, acto administrativo, cláusula de convenio colectivo, pacto individual, decisiones unilaterales del empleador y todo acto que contenga o suponga cualquier perjuicio o beneficio cuya causa sea el ejercicio de la libertad sindical. A ello, se le ha agregado una tutela específica para los más expuestos: los dirigentes sindicales, que se expresa sintéticamente en la consagración del instituto del despido-propuesta, es decir, que para que opere la extinción del vínculo laboral se requiere un proceso judicial previo en el que el empleador pruebe la comisión de la falta grave imputada.

Como la descentralización productiva ha hecho explotar el paradigma de la empresa unitaria afectando gravemente a los países como el Perú, cuyo escenario de actuación sindical era justamente aquella, la necesidad constitucional de garantizar y fomentar la tutela colectiva (artículo 28) conduce a acompañar las nuevas posibilidades organizativas (que involucran a grupos de empresas, redes de empresas o sectores, de actividad, etcéter) con garantías efectivas para la negociación en tales ámbitos. En función de ello, señalamos que en el artículo 343 de la LGT propuesta se establece que la falta de acuerdo sobre el nivel de la negociación colectiva (inicial o por cambio de uno preexistente) se resolverá por medio de un arbitraje. Asimismo, se precisan dos temas clave de la negociación colectiva: el deber de negociar de buena fe (y sus seis obligaciones derivadas) y el derecho de información (con plazos de entrega previos a la elaboración del pliego de peticiones), cuya deficiente regulación vigente les ha restado efectividad actualmente.

Además de ello, se ha establecido de la manera más amplia posible el recurso al arbitraje potestativo, es decir, a iniciativa de una de las partes, como expresión del mandato constitucional de promoción de las formas pacíficas de solución de conflictos colectivos, que ya venía señalándose en la jurisprudencia del Tribunal Constitucional e incluso, recientemente, del Poder Judicial, a partir de lo previsto en la regulación actual. Este recurso a la heterocomposición del conflicto colectivo tiene a su vez una gran legitimidad social, al haber sido consensuada por

EL MODELO DE RELACIONES COLECTIVAS

PERUANO: DEL INTERVENCIONISTA Y RESTRICTIVO AL PROMOCIONAL

THE MODEL OF

PERUVIAN

COLLECTIVE

RELATIONS: FROM INTERVENTIONIST AND RESTRICTIVE TO PROMOTIONAL 
empleadores y trabajadores cuando trataron la propuesta inicial (2002) de LGT, y tiene especial relevancia en un momento en que la debilidad sindical conduce a dejar abiertos por años los procesos de negociación colectiva al no poder recurrir eficazmente a la huelga.

Y, finalmente, en el ámbito de la regulación del derecho de huelga también encontramos regulaciones del proyecto de LGT que calzan perfectamente con la obligación constitucional de fomento de la negociación colectiva. Entre las más relevantes está la expulsión del proceso de calificación de la legalidad de las huelgas del ámbito del Ministerio de Trabajo, para residenciarlo en un órgano tripartito creado en el seno del Consejo Nacional del Trabajo, que tendrá a su cargo además los conflictos de divergencia sobre la dimensión de los servicios mínimos en los casos de servicios esenciales o de mantenimiento. Asimismo, pueden contarse en el terreno promocional la derogación de la norma que prohibía las modalidades atípicas de huelga, la regulación de los servicios esenciales respetando lo previsto por la OIT al respecto, y la imposición del pago de las remuneraciones y otros derechos dejados de pagar durante la huelga cuando judicialmente queda demostrado que la causa de la medida fue un incumplimiento empresarial.

Para concluir con este breve resumen de la línea promocional del proyecto de LGT, está el plano colateral, con el que se ha abierto un nuevo camino: el de permitir que ciertas restricciones legales puedan ser superadas por la vía de la negociación colectiva. Es el caso del límite máximo del 20\% de trabajadores que pueden contratarse a tiempo parcial, establecido por el artículo 29, que puede ampliarse por convenio colectivo, con lo que se le da a la negociación colectiva un rol organizacional (en terminología de la doctrina norteamericana) que puede resultar muy atractivo para la marcha de la empresa.

De todo lo dicho se puede concluir que el fomento de la negociación colectiva es en realidad la piedra de toque, el eje, la filosofía que explica la propuesta legislativa bajo comentario, perfeccionando la propuesta inicial de 2002 y contando con una legitimidad no solo jurídica sino social e internacional indudable, que de aprobarse marcará un hito en la consolidación del Estado Constitucional de Derecho en el Perú. 